\title{
Can Antipsychotics Improve Social Cognition in Patients with Schizophrenia?
}

\author{
Katarzyna Kucharska-Pietura • Ann Mortimer
}

Published online: 27 March 2013

(C) The Author(s) 2013. This article is published with open access at Springerlink.com

\begin{abstract}
Social cognition is described as the higher mental processes that are engaged while people store, process, and use social information to make sense of themselves and others. Aspects of social cognition include emotion perception, social cue interpretation, attribution style, and theory of mind, all of which appear disordered in schizophrenia. Such social cognitive deficits are believed to be important predictors of functional outcome in schizophrenia, therefore they may represent a crucial treatment target. Few studies have evaluated the influence of antipsychotic treatment on these deficits. The purpose of this review is to examine the relationship between antipsychotic treatment and social cognition, whether antipsychotics improve social cognitive function, and if so to explore differential medication effects. Comprehensive searches of PsycINFO and MEDLINE/PUBMED were conducted to identify relevant published manuscripts. Fifteen relevant papers published in English were found, describing original studies. On the basis of this review, we have drawn the following conclusions: first, the results do not engender optimism for the possibility that antipsychotic drugs can specifically facilitate social recovery. Second, the actions of
\end{abstract}

K. Kucharska-Pietura · A. Mortimer

NAViGO, Grimsby, UK

e-mail: A.M.Mortimer@hull.ac.uk

K. Kucharska-Pietura

Hull York Medical School, Hull, UK

K. Kucharska-Pietura ( $\square)$

Rharian Fields, The Gardens, Diana Princess of Wales Hospital, Grimsby DN33 2BA, UK

e-mail: kate.kucharska-pietura@nhs.net

A. Mortimer

University of Hull, Hull, UK antipsychotics on social cognition are inconclusive, due to lack of standardization across research groups, leading to inconsistencies between study designs, methods used, and medication dosages. Third, large-scale longitudinal investigations are needed to explore the unclear relationships between social cognition, symptoms, and functional outcome. Other non-pharmacological treatments focusing on training patients in the social cognitive areas may hold more promise.

\section{Introduction}

Social cognition has been defined as the way we perceive, interpret, and understand social information [1] or as "the processes that allow a person to understand, act on, and benefit from the interpersonal world" [2]. A further overall definition was given by Adolphs [3] who described social cognition as "the ability to construct representations of the relation between oneself and others and to use those representations flexibly to guide social behaviour."

Aspects of social cognition include emotion perception, social cue interpretation, attribution style, and theory of mind. Affect perception is the ability to infer emotional information, in other words what a person is feeling, presented either in visual or auditory form. Social cue perception refers to a person's ability to ascertain social cues from behaviour provided in a social context, and refers to a person's comprehension of social rules [4]. Attribution style, known as a personalizing bias, refers to an individual's own perception and interpretation of facts and events [5]. The attribution of mental states, such as desires, intentions, and beliefs, to other people has been referred to as "theory of mind" (ToM) or "mentalising" $[6,7]$. ToM involves both the ability to understand that others have 
mental states different from one's own, and the capability to make correct inferences about the content of those mental states [5].

The neurophysiological and neurochemical underpinnings of social cognition in schizophrenia are a scientific domain that requires further exploration. Several neurotransmitters seem to play a considerable role in social cognitive processes, and their circuitries are deemed to be altered in schizophrenia. The hypothalamic peptides arginine vasopressin (AVP) and oxytocin (OXT) have been described as social hormones that may mediate social behaviour [8] including social motivation, approach behaviour [9], and ToM [10, 11]. Recently, abnormal oxytocinergic and dopaminergic signalling in the amygdalae has been proposed to explain dysfunction in the social cognitive domain in schizophrenia [12].

Serotonin is another neurotransmitter linked to social behaviour, including roles in cognition, mood, and aggression, alongside motivation, energy levels, and sleep $[13,14]$. There is increasing interest in the correlation between negative symptoms of schizophrenia and abnormal neurotransmission at serotonin $5-\mathrm{HT}_{2}$ receptors [15].

Dopamine appears as a key neurotransmitter in the aetiopathogenesis of schizophrenia described as crucially involved in the attribution process as well as emotional perception, giving not only meaning but also salience to the objects in our environment $[16,17]$. Deregulation of the dopaminergic system leads to the production of dopamine regardless of incoming stimuli, which results in giving meaning to their meta-representations, thereby creating a misguided inner reality of actually meaningless objects. This maladaptive attribution system created during psychosis is very often implicated permanently in the patient's experience, regardless of pharmacological blocking of excess dopamine [16].

A growing body of literature has shown consistently that schizophrenia patients compared with healthy controls present with social cognitive impairments that are relatively stable and persistent, suggesting that it is a trait-dependent rather than state-dependant aspect of the disorder [18-20]. These deficits have been widely described as modifying patients' behaviour when interacting with other people (ToM deficits) [7, 20-22] and in recognizing emotions [23$25]$ and other social information cues $[18,26]$. Therefore, social cognitive deficits are believed to be important predictors of functional outcome in schizophrenia [5, 27]. Such deficits represent an obvious substrate for treatment in schizophrenia.

Pharmacological treatment research on social cognition in schizophrenia has been relatively limited: recent data on the effects of second- and first-generation antipsychotics (SGA/FGA) on various domains of social cognition remain inconclusive.

This paper aims (1) to appraise current evidence on the impact of antipsychotics upon social cognitive functioning in schizophrenia, to find out if antipsychotics do really improve social cognitive functions; and if yes, (2) to explore differential medication effects on social cognition, if any.

A comprehensive search of the PsycINFO and MEDLINE/PUBMED databases for articles in English published till 31 December 2012 was conducted. Within the domain of social cognition, the following search terms were used: emotion/affect perception, emotion/affect recognition, attribution/attributional style, theory of mind/mentalising, social cognition, social competence, and social cue perception. Within the domain of psychopharmacology outcome, the following terms were used: conventional antipsychotics, atypical antipsychotics, atypicals, and clinical trials.

Search terms for schizophrenia included the following: psychosis, schizophrenia, and schizoaffective disorder.

\subsection{Search Strategy}

The following search keywords were used:

1. schizophrenia AND social cognition AND antipsychotics; 224 articles, 15 utilised

2. schizophrenia AND emotion perception AND antipsychotics; 63 papers, 6 utilised

3. schizophrenia AND facial affect AND antipsychotics; 29 papers, 4 utilised

4. schizophrenia AND theory of mind AND antipsychotics; 14 papers, 3 utilised

5. schizophrenia AND attribution AND antipsychotics; 20 articles, 0 utilised

6. schizophrenia AND attributional style AND antipsychotics; 3 articles, 1 utilised

7. schizophrenia AND social competence AND antipsychotics; 33 articles, 2 utilised

8. schizophrenia AND social cue perception AND antipsychotics; 2 papers, 1 utilised

\subsection{Inclusion Criteria}

The papers were utilised in the current review if they were written in English and had reported experimental studies of aspects of social cognition in schizophrenia treated with antipsychotic medication. Although 32 papers were identified there was much overlap between the results of individual searches: 15 papers in total were accrued from all eight searches and are reviewed here (see Table 1). 


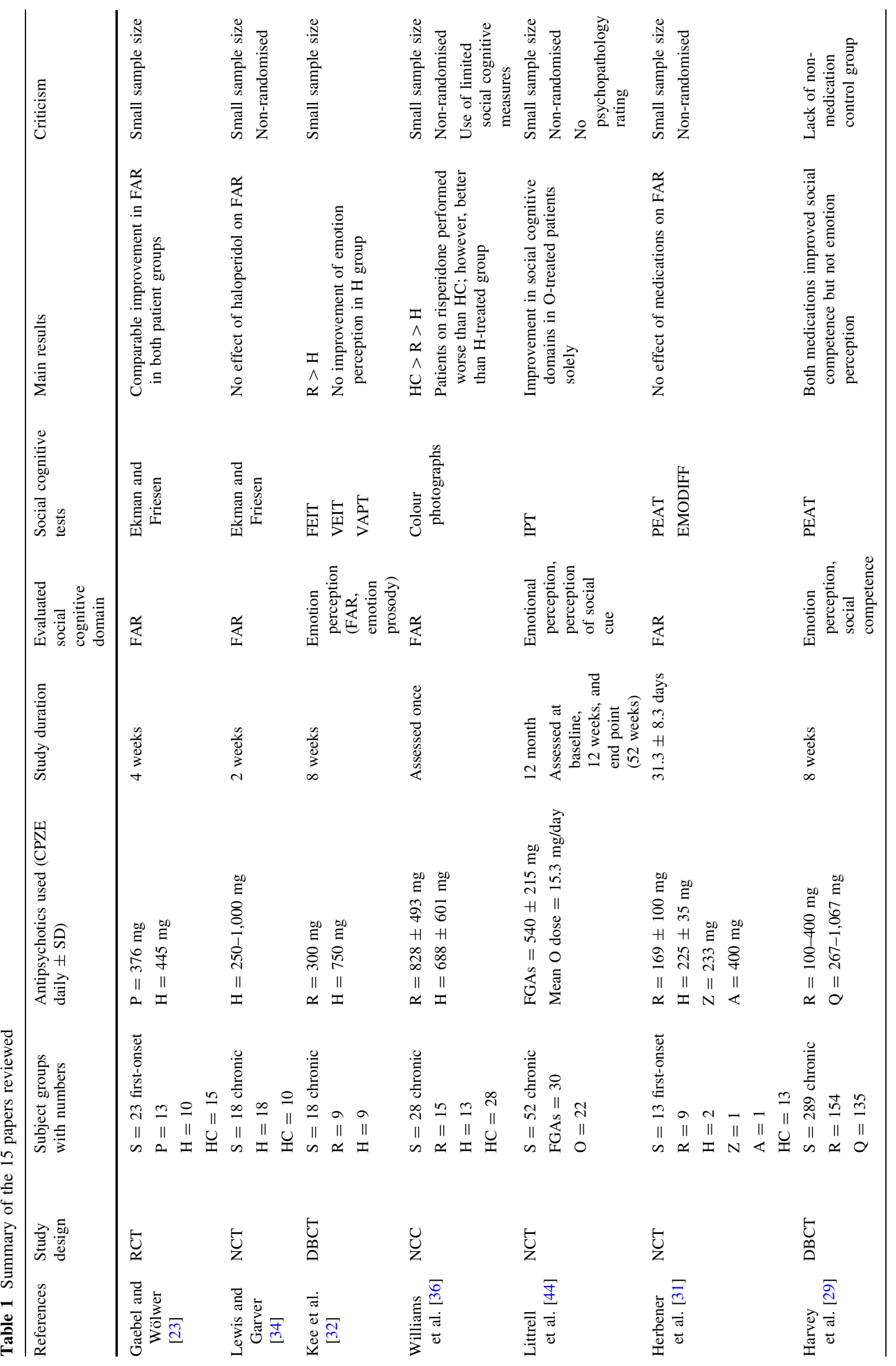




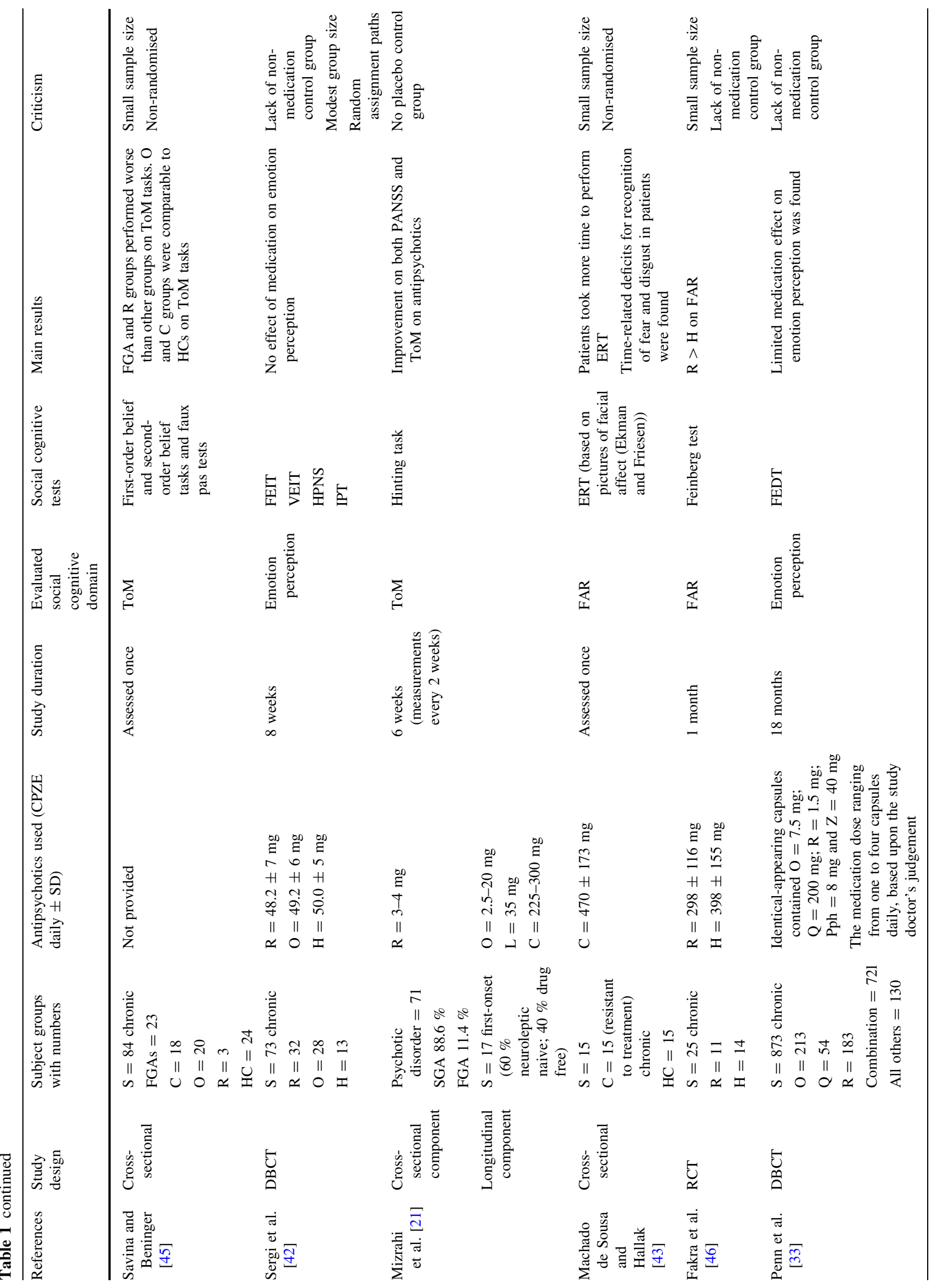




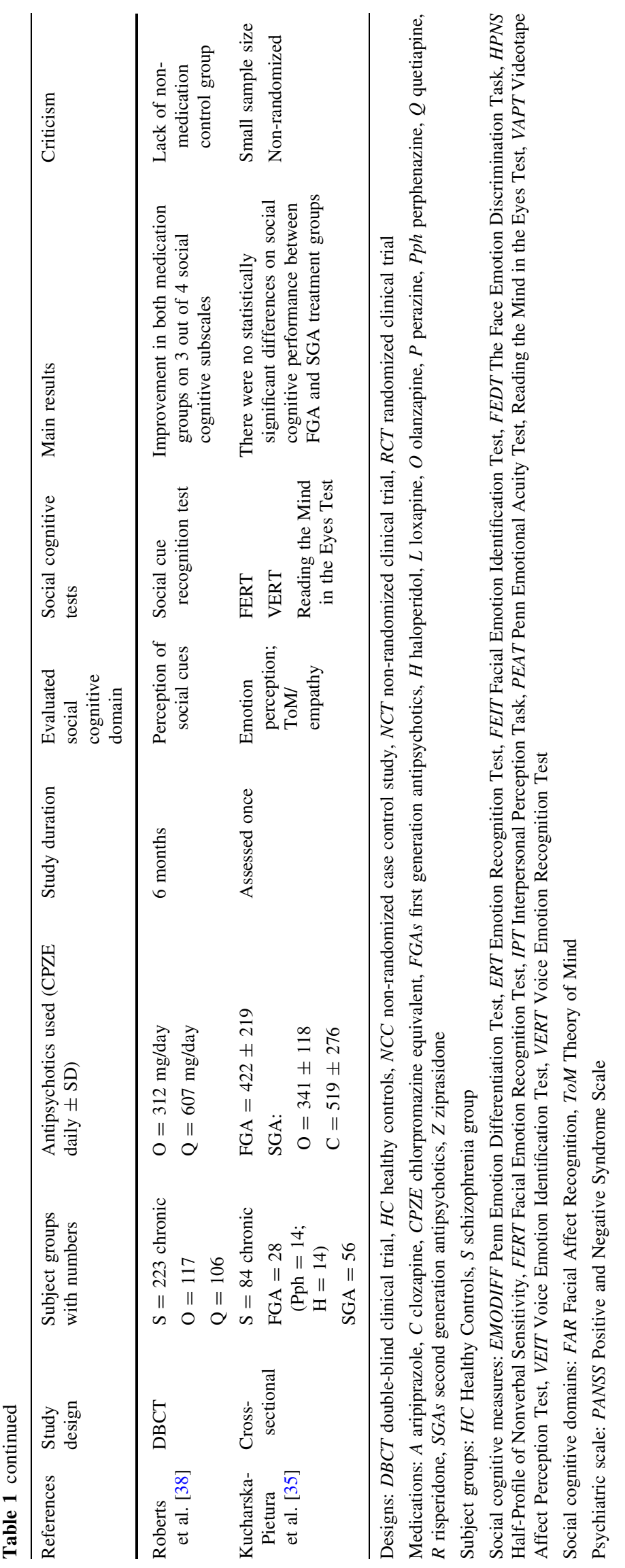




\section{The Place of Social Cognitive Deficit in Multifactorial Models of Schizophrenia: Symptom, or Neurocognitive Compromise?}

The analysis of emotional behaviour in schizophrenia is fundamental to the notion of dementia praecox introduced by Kraepelin, and a question fielded by Bleuler on the basis of 'Affektivität': "What happened to feelings in dementia praecox?" This has constituted and still constitutes a scientific challenge [28]. While symptom-based approaches have understandably dominated most aspects of pharmacological intervention in schizophrenia research, a limited number of studies have investigated the effect of symptoms on social cognition [29-33].

To the best of the authors' knowledge, the majority of studies fail to demonstrate a clear relationship between overall symptom severity scores and performance on social cognitive measures [29, 34-36].

However, there are suggestions that negative and disorganized symptoms may be related to social cognitive functioning [21, 23, 31, 37, 38].

Relationships among social cognitive constructs and negative symptoms are, however, not clear. Although some overlap exists between negative symptoms and social cognition in schizophrenia, according to participants at a National Institute of Mental Health (NIMH) conference where this issue was addressed [39] it is unwise to combine the constructs at this point in time. The consensus was that it is more informative to study negative symptoms and social cognition separately and to analyse relationships between them, until we know more about areas of convergence and divergence. Regarding positive symptoms [32], there has been some linkage between attributional style and paranoid delusions [40]. There is virtually no literature that has developed the relationship between disorganisation symptoms and social cognition.

Penn et al. [1] argued that multifactorial models of schizophrenia, including only non-social cognitive processes i.e. 'neurocognition', did not adequately explain the social functioning impairment in schizophrenia. Subsequently, social cognition was seen as a key domain for consideration during the first meeting of the NIMH-sponsored Measurement and Treatment Research to Improve Cognition in Schizophrenia (MATRICS) initiative [27] and it was ultimately included as one of the seven domains represented in the MATRICS Consensus Cognitive Battery for clinical trials in schizophrenia [39].

Therefore, current accepted wisdom is that social cognitive deficit is a variety of neurocognitive compromise; it does not represent a symptom or group of symptoms by itself. The development of objective tests to quantify degrees of social cognitive impairment argues further for the validity of this construct.

\section{Does Antipsychotic Treatment Improve Social Cognition?}

Lewis and Garver [34] assessed facial affect recognition in 18 patients on haloperidol (5-20 mg/day) compared with 10 healthy controls in the course of their 2-week nonrandomised clinical trial. An impairment in facial affect recognition was found in the schizophrenia group. This deficit was not related to psychopathology symptom scores.

Bellack et al. [41] assessed the effects of clozapine and risperidone on social skills at baseline, week 17, and week 29 in patients with schizophrenia using the Maryland Assessment of Social Competence. No significant medication effect on social competence was found despite clinical improvement on both medications. Similarly, Herbener et al. [31] described no beneficial effect of antipsychotics after 1 month (nine patients on risperidone) regarding facial affect recognition in 13 patients with firstepisode psychosis.

It is worth noting that these studies were not adequately powered to draw definite conclusions. However, two influential randomised studies failed again to support the hypothesis that antipsychotics improve social cognition [29, 42]. Harvey et al. [29] found that patients with schizophrenia treated with either risperidone ( $n=154 ; 2-8 \mathrm{mg} /$ day $)$ or quetiapine ( $n=135 ; 200-800 \mathrm{mg} /$ day) for an 8 -week period of double-blind treatment did not improve their facial affect recognition, using the Penn Emotional Acuity Test. Similarly, Sergi et al. [42] found no evidence of treatmentrelated differences in social cognition in 73 patients with a diagnosis of schizophrenia in an 8-week double-blind study of risperidone, olanzapine, and haloperidol. Interestingly, when the potential influence of changes in neurocognition was statistically controlled for, there was no within-group change in social cognition. This suggests that social cognition and neurocognition are not the same thing, in other words, they vary independently of each other.

Alternatively, Gaebel and Wölwer [23] and Roberts et al. [38] demonstrated, respectively, significant improvement in facial affect recognition in patients on FGAs (haloperidol or perazine) and on the Social Cue Recognition Test in patients treated with olanzapine $(n=117)$ or quetiapine ( $n=106$ ) [Table 1]. Similarly Mizrahi et al. [21] studied 17 drug-free patients who then received antipsychotic treatment for 6 weeks: the effect on psychotic symptoms and ToM, using a hinting task, was measured every 2 weeks. The hinting task score was associated with negative and general symptom scores. Both the Positive and Negative Syndrome Scale (PANSS) positive scores and ToM improved after medication was started, particularly during the first 2 weeks of antipsychotic treatment.

Surprisingly, Machado de Sousa and Hallak [43] reported no differences in recognition accuracy or 
emotional intensity scores within the Facial Emotion Recognition Task between patients on clozapine compared with healthy controls. Since clozapine is a superior antipsychotic drug, this suggests that clozapine treatment may have corrected any deficit. The analysis of individual emotions, however, demonstrated a specific time-related deficit affecting the recognition of fear and disgust. Moreover, Harvey et al. [29] reported a similar apparent differential effect in patients treated for 8 weeks with quetiapine or risperidone: emotion perception remained unchanged, whereas social competence improved. This correlated with concurrent improvement in other aspects of neuropsychological performance, such as executive function and memory.

\subsection{Is There Any Differential Effect on Social Cognition Between Antipsychotic Agents?}

A number of influential studies have confirmed that SGAs outperformed FGAs in a range of clinical efficacy parameters, including the domain of social cognition [32, 36, 4446]. It has been argued that SGAs' strong affinity for $5-\mathrm{HT}_{2}$ receptors [14] via the disinhibitory effect of serotonin antagonism on dopamine release in the prefrontal area may eventually improve emotion perception and social functioning [14]. However, both FGAs and SGAs also affect dopamine regulation in the mesocorticolimbic system, which suggests the potential for regulation of the amygdalae as an emotional manager [17].

Furthermore, clozapine and olanzapine increased dopamine outflow in the medial prefrontal cortex (mPFC), but not in the striatum or nucleus accumbens, whereas haloperidol had no effect in the mPFC but increased dopamine outflow in the striatum [47].

As a rule of thumb, frontal dopamine deficiency, perhaps as a response to striatal overactivity, has been considered germane to the induction of negative symptoms, associated with cognitive deficit and impaired social cognition. Therefore, trials have investigated the differential effects of FGAs and SGAs upon social cognition.

Kee et al. [32] evaluated the ability to recognise "emotional" faces in 20 treatment-resistant patients at baseline and after 8 weeks of treatment with risperidone or haloperidol, in a double-blind trial. The results of this study confirmed the positive influence of treatment with risperidone on the performance of facial affect tasks. Williams et al. [36] later reported similarly, that schizophrenic patients on haloperidol underperformed those on risperidone and healthy controls in recognising facial emotional expressions. Haloperidol-treated patients showed reduced fixation (attention) to salient features for neutral and happy expressions whereas risperidone-treated subjects and healthy controls achieved comparable results, displaying significantly better fixation to salient features for these expressions. This was followed by Littrell et al. [44] who in an open study found that 22 schizophrenia patients treated with olanzapine for 12 months performed better on a social perception measure, the Interpersonal Perception Task, than 30 patients on FGAs.

Fakra et al. [46] reported that 25 acute schizophrenia patients randomised to risperidone performed a facial affect discrimination task significantly better than those treated with haloperidol after 4 weeks. It was concluded that risperidone may specifically act on the processing of emotionladen information: findings could not be explained on the grounds of facial recognition alone. However, Savina and Beninger [45] demonstrated that ToM performance in schizophrenia patients was related to maintenance, rather than acute treatment effects: they suggested that olanzapine and clozapine, but not risperidone or FGAs, may improve or protect ToM ability in this scenario.

It is worth mentioning that none of the studies above, apart from that of Harvey et al. [29], was both randomised and adequately powered. The lack of standardised social cognitive measures coupled with psychopathology rating scales detracts from their value. Common sense dictates that the active, distressing symptoms of acutely ill patients and the far from optimal state of arousal that these induce must seriously impair performance of any cognitive task that requires optimal attention and concentration. 'Control' tasks, to uphold the specificity of any improvement in social cognition, are conspicuous by their absence.

Consistent with the conclusion that these positive findings may be more apparent than real is a substantial body of literature reflecting far fewer differences between atypical and conventional antipsychotic drugs than initially suggested [48].

Of enormous influence is the CATIE trial (Clinical Antipsychotic Trials for Intervention Effectiveness trial), which failed to demonstrate differential antipsychotic effects on social cognition. To wit, Penn et al. [33] assessed emotion perception in 873 CATIE patients randomised to quetiapine, olanzapine, risperidone, ziprasidone (all SGAs), or perphenazine (FGA). Patients completed the Face Emotion Discrimination Task [49] immediately prior to randomisation and after 2 months of treatment. At baseline, $60 \%$ of participants were on a SGA, $15 \%$ on a FGA, and $25 \%$ of subjects were antipsychotic free. Non-statistically significant improvement in emotion perception at 2 months was observed: the treatment groups did not differ from one another.

Finally, Kucharska-Pietura et al. [35] assessed deficits in social cognitive functioning in a naturalistic pragmatic sample of partially remitted stable schizophrenia inpatients, 28 being treated with a FGA (perphenazine or haloperidol), 56 being treated with a SGA (olanzapine or 
clozapine), and 50 healthy controls. In line with previous findings, there were no differences between the patient groups in emotional perception and ToM/empathy. This is particularly striking given the supposedly superior effects of clozapine previously reported. There were small but significant advantages for SGAs in non-social low-level visual processing: this was thought to result from SGAs' weaker antagonism of dopamine receptors in the retina [35].

\section{Conclusions}

First, overall, antipsychotic drugs of either class demonstrate little reliable effect upon social cognition [38, 50]. There is a modicum of support for the use of oxytocin as an adjunct to antipsychotic drugs [11] but whether this latest finding is a valid effect remains a matter of conjecture. By contrast, recent randomized intervention studies of specialised psychosocial treatment programmes for social cognition report very promising results in the improvement of emotional perception and social skills in schizophrenia [50-52].

Secondly, the literature suffers from inconsistencies in study design, particularly a prevalence of non-randomised approaches based upon cross-sectional assessments, which do not reflect the later NIMH recommendations. Nor are medication doses standardised. Most sample sizes are quite small, and there is inadequate control of pertinent clinical variables. This overlaps with three obstacles to research progress identified by the NIMH group: (1) psychometrics and measurement, (2) maturity of the field, and (3) a lack of interdisciplinary bridges between clinical and basic researchers [39].

Finally, large-scale longitudinal investigations are needed to explore the unclear relationships between social cognition, symptoms, and functional outcome. If social cognition proves to represent a neurocognitive construct, we suspect related to premorbid personality, then it is not logical to expect current antipsychotic treatments designed to attenuate active symptoms to have any significant effect other than through symptom control, thus abolishing the 'noise' of symptoms in the patient's attempts at social cognitive function. Other treatments, quite possibly training patients in the areas in which they are impaired, may hold more promise.

Acknowledgments The authors report no conflicts of interest in this work. No sources of funding were received to prepare this article.

Open Access This article is distributed under the terms of the Creative Commons Attribution Noncommercial License which permits any noncommercial use, distribution, and reproduction in any medium, provided the original author(s) and the source are credited.

\section{References}

1. Penn DL, Corrigan PW, Bentall RP, et al. Social cognition in schizophrenia. Psychol Bull. 1997;121:114-32.

2. Corrigan PW, Penn DL, editors. Social cognition and schizophrenia. Washington, DC: American Psychological Association; 2001.

3. Adolphs R. The neurobiology of social cognition. Curr Opin Neurobiol. 2001;11:231-9.

4. Bigelow N, Paardiso S, Adolphs R, et al. Perception of social stimuli in schizophrenia. Schizophr Res. 2006;83:257-67.

5. Couture SM, Penn DL, Roberts DL. The functional significance of social cognition in schizophrenia: a review. Schizophr Bull. 2006;32(Suppl 1):S44-63.

6. Brüne M. Emotion recognition, 'theory of mind', and social behaviour in schizophrenia. Psychiatry Res. 2005;133:135-47.

7. Brüne M, Schaub D. Mental state attribution in schizophrenia: what distinguishes patients with "poor" from patients with "fair" mentalising skills? Eur Psychiatry. 2012;27:358-64.

8. Ebstein RP, Knafo A, Mankuta D, et al. The contributions of oxytocin and vasopressin pathway genes to human behavior. Horm Behav. 2012;61:359-79.

9. Domes G, Heinrichs M, Gläscher J, et al. Oxytocin attenuates amygdala responses to emotional faces regardless of valence. Biol Psychiatry. 2007;62:1187-90.

10. Domes G, Heinrichs M, Michel A, et al. Oxytocin improves "mind-reading" in humans. Biol Psychiatry. 2007;61:31-3.

11. Pedersen CA, Gibson CM, Rau SW, et al. Intranasal oxytocin reduces psychotic symptoms and improves Theory of Mind and social perception in schizophrenia. Schizophr Res. 2011;132:50-3.

12. Rosenfeld AJ, Lieberman JA, Jarskog LF. Oxytocin, dopamine, and the amygdala: a neurofunctional model of social cognitive deficits in schizophrenia. Schizophr Bull. 2011;37:1077-87.

13. Ichikawa $\mathrm{J}$, Ishii $\mathrm{H}$, Bonaccorso $\mathrm{S}$, et al. 5-HT(2A) and $\mathrm{D}(2)$ receptor blockade increases cortical DA release via 5-HT(1A) receptor activation: a possible mechanism of atypical antipsychotic-induced cortical dopamine release. J Neurochem. 2001; 76:1521-31.

14. Kapur S, Remington G. Atypical antipsychotics: new directions and new challenges in the treatment of schizophrenia. Annu Rev Med. 2001;52:503-17.

15. Akhondzadeh S. The 5-HT hypothesis of schizophrenia. IDrugs. 2001;4:295-300.

16. Kapur S. Psychosis as a state of aberrant salience: a framework linking biology, phenomenology, and pharmacology in schizophrenia. Am J Psychiatry. 2003;160:13-23.

17. Salgado-Pineda P, Delaveau P, Blin O, et al. Dopaminergic contribution to the regulation of emotional perception. Clin Neuropharmacol. 2005;28:228-37.

18. Addington J, Addington D. Social and cognitive functioning in psychosis. Schizophr Res. 2008;99:176-81.

19. Kucharska-Pietura K, David AS, Masiak M, et al. Perception of facial and vocal affect by people with schizophrenia in early and late stages of illness. Br J Psychiatry. 2005;187:523-8.

20. Sprong M, Schothorst P, Vos E, et al. Theory of mind in schizophrenia: meta-analysis. Br J Psychiatry. 2007;191:5-13.

21. Mizrahi R, Korostil M, Starkstein SE, et al. The effect of antipsychotic treatment on Theory of Mind. Psychol Med. 2007;37: 595-601.

22. Roncone R, Falloon IR, Mazza M, et al. Is theory of mind in schizophrenia more strongly associated with clinical and social functioning than with neurocognitive deficits? Psychopathology. 2002;35:280-8.

23. Gaebel W, Wölwer W. Facial expression and emotional face recognition in schizophrenia and depression. Eur Arch Psychiatry Clin Neurosci. 1992;242:46-52. 
24. Edwards J, Pattison PE, Jackson HJ, et al. Facial affect and affective prosody recognition in first-episode schizophrenia. Schizophr Res. 2001;48:235-53.

25. Trémeau F. Emotion deficits in schizophrenia. Dial Clin Neurosci. 2006;8:58-68.

26. Penn DL, Ritchie M, Francis J, et al. Social perception in schizophrenia: the role of context. Psychiatry Res. 2002;109: 149-59.

27. Green MF, Nuechterlein KH, Gold JM, et al. Approaching a consensus cognitive battery for clinical trials in schizophrenia: the NIMH-MATRICS conference to select cognitive domains and test criteria. Biol Psychiatry. 2004;56:301-7.

28. Bleuler EP. Affektivität, Suggestibilität. Halle: Paranoi; 1906.

29. Harvey PD, Patterson TL, Potter LS, et al. Improvement in social competence with short-term atypical antipsychotic treatment: a randomized, double-blind comparison of quetiapine versus risperidone for social competence, social cognition, and neuropsychological functioning. Am J Psychiatry. 2006;163:1918-25.

30. Hempel RJ, Dekker JA, Van Beveren NJ, et al. The effect of antipsychotic medication on facial affect recognition in schizophrenia: a review. Psychiatry Res. 2010;178:1-9.

31. Herbener ES, Hill K, Marvin RW, et al. Effects of antipsychotic treatment on emotion perception deficits in first-episode schizophrenia. Am J Psychiatry. 2005;162:1746-8.

32. Kee KS, Kern RS, Marshall BD, et al. Risperidone versus haloperidol for perception of emotion in treatment-resistant schizophrenia: preliminary findings. Schizophr Res. 1998;31:159-65.

33. Penn DL, Keefe RS, Davis SM, et al. The effects of antipsychotic medications on emotion perception in patients with chronic schizophrenia in the CATIE trial. Schizophr Res. 2009;115: $17-23$.

34. Lewis SF, Garver DL. Treatment and diagnostic subtype in facial recognition in schizophrenia. J Psychiatry Res. 1995;29:5-11.

35. Kucharska-Pietura K, Mortimer A, Tylec A, et al. Social cognition and visual perception in schizophrenia inpatients treated with first-and second-generation antipsychotic drugs. Clin Schizophr Relat Psychoses. 2012;6:14-20.

36. Williams LM, Loughland CM, Green MJ, et al. Emotion perception in schizophrenia: an eye movement study comparing the effectiveness of risperidone vs. haloperidol. Psychiatry Res. 2003;120:13-27.

37. Brekke JS, DeBonis JA, Graham JW. A latent structure analysis of the positive and negative symptoms in schizophrenia. Compr Psychiatry. 1994;35:252-9.

38. Roberts DL, Penn DL, Corrigan P, et al. Antipsychotic medication and social cue recognition in chronic schizophrenia. Psychiatry Res. 2010;178:46-50.
39. Green MF, Penn DL, Bentall R, et al. Social cognition in schizophrenia: an NIMH workshop on definitions, assessment, and research opportunities. Schizophr Bull. 2008;34:1211-20.

40. Combs DR, Penn DL, Michael CO, et al. Perceptions of hostility by persons with and without persecutory delusions. Cogn Neuropsychiatry. 2009;14:30-52.

41. Bellack AS, Schooler NR, Marder SR, et al. Do clozapine and risperidone affect social competence and problem solving? Am J Psychiatry. 2004;161:364-7.

42. Sergi MJ, Green MF, Widmark C, et al. Social cognition [corrected] and neurocognition: effects of risperidone, olanzapine, and haloperidol. Am J Psychiatry. 2007;164:1585-92.

43. Machado de Sousa JP, Hallak JE. Neurocognitive functioning and facial affect recognition in treatment-resistant schizophrenia treated with clozapine. Schizophr Res. 2008;106:371-2.

44. Littrell KH, Petty RG, Hilligoss NM, et al. Improvement in social cognition in patients with schizophrenia associated with treatment with olanzapine. Schizophr Res. 2004;66:201-2.

45. Savina I, Beninger RJ. Schizophrenic patients treated with clozapine or olanzapine perform better on theory of mind tasks than those treated with risperidone or typical antipsychotic medications. Schizophr Res. 2007;94:128-38.

46. Fakra E, Salgado-Pineda P, Besnier N, et al. Risperidone versus haloperidol for facial affect recognition in schizophrenia: findings from a randomised study. World J Biol Psychiatry. 2009;10: $719-28$.

47. Heidbreder CA, Foxton R, Cilia J, et al. Increased responsiveness of dopamine to atypical, but not typical antipsychotics in the medial prefrontal cortex of rats reared in isolation. Psychopharmacol Berl. 2001;156:338-51.

48. Fakra E, Kaladjian A, Adida M, et al. Schizophrenia, psychotropic drugs and cognition. Encephale. 2011;37(Suppl 2):S137-42.

49. Kerr SL, Neale JM. Emotion perception in schizophrenia: specific deficit or further evidence of generalized poor performance? J Abnorm Psychol. 1993;102:312-8.

50. Sachs G, Winklbaur B, Jagsch R, et al. Training of affect recognition (TAR) in schizophrenia-impact on functional outcome. Schizophr Res. 2012;138:262-7.

51. Combs DR, Adams SD, Penn DL, et al. Social cognition and interaction training (SCIT) for inpatients with schizophrenia spectrum disorders: preliminary findings. Schizophr Res. 2007; 91:112-6.

52. Roberts DL, Penn DL. Social cognition and interaction training (SCIT) for outpatients with schizophrenia: a preliminary study. Psychiatry Res. 2009;166:141-7. 